\title{
High frequency impulse ground penetrating radar application in assessment of interlayer connections
}

\author{
Jacek Sudyka ${ }^{1,}{ }^{*}$, Lech Krysiński ${ }^{1}$, Adam Zofka ${ }^{1}$, Marek Pszczoła ${ }^{2}$, and Piotr Jaskuła ${ }^{2}$ \\ ${ }^{1}$ Road and Bridge Research Institute, Warsaw 03-302, Poland \\ ${ }^{2}$ Gdansk University of Technology, Gdansk 80-233, Poland
}

\begin{abstract}
Ground Penetrating Radar (GPR) technique is commonly used in the nondestructive evaluation of pavement structures. In particular, this method is used to estimate thicknesses of pavement layers as well as it can be utilized in advanced studies of pavement structures. The device presented in this paper comprise the high frequency impulse antennas that allow for investigating the interlayer zones in terms of their electromagnetic properties (e.g. dielectric constant). In some cases these electromagnetic responses can be suitable in the assessment of layer bonding in the pavement structure. This paper discusses the assessment of the quality of asphalt pavement interlayer bonding with the use of high frequency GPR techniques. The preliminary laboratory measurements were performed using an impulse antenna in the zero-offset configuration combined with the large-scale models simulating an idealized horizontal delamination. These measurements allowed to estimate the antenna sensitivity to detect interlayer connection under dry and wet conditions. Analysis of collected results led to formulating practical conclusions regarding critical limitations of the measuring system and adequate methods of signal processing and interpretation.

The field investigations consisted of the GPR measurements along selected road sections and collection of the core samples at the locations associated with the specific reflexes. Inspection of the cores provided some real insights into the structure of different delaminations associated with characteristic reflexes. Analysis showed the reflection properties are able to expresses some important features of the interlayer zone, such as delaminations, presence of alien material at the interface, insufficient compaction occurring at the base of layer, and water penetration.
\end{abstract}

\section{Introduction}

The bonding of asphalt layers is a significant factor that directly influences the strength and durability of pavement structures. Due to insufficient bonding between asphalt layers, individual layers can move horizontally independently from each other that weakens the entire structure and leads to premature surface and structural failures [1]. In particular,

\footnotetext{
* Corresponding author: jsudyka@ibdim.edu.pl
} 
debonded asphalt layers can lead to corrugation, slippage and transverse cracking of pavement surfaces that significantly affect traffic safety [2]. In the case of weak bonding predicted pavement life may drop to as low as $40 \%$ [3]. According to Judycki [4] fatigue life decreases rapidly when the interlayer connection is weakened. When there is a total lack of bonding the fatigue life of pavement decreases several times. While such an extreme condition is unlikely to occur, even with the partial bonding of 50\% the decrease in fatigue life is clearly evident. In another study, Raab and Partl [2] estimated that a defective connection between asphalt layers may, in extreme cases, reduce the expected fatigue life of pavement by $30 \%$. According to Raposerias and Castro-Fernas [5] the combined effect of high temperature and traffic can improve the interlayer connection provided however that the bond has not been totally disconnected. Vaitkus [6] has demonstrated that insufficient bonding between asphalt layers can cause corrugation and rutting of pavement. He states that cracks in asphalt pavement occur because of insufficient bonding of asphalt layers and reports also that an optimal bonding is necessary in order to obtain a sufficient asphalt pavement strength to prevent rutting. Raposeiras [7] have determined that the deformation of asphalt pavement layers increases significantly if the layer bonding decreases from $100 \%$ to $90 \%$, which results in a premature deterioration of asphalt pavement.

There are three types of asphalt layer bonding [8]:

- sufficiently bonded - asphalt layers act as a monolithic structure. A large shear stress is created and no deformations (displacements) are developed,

- partially bonded - depends on the interlocking strength. The shear stress and deformations (displacements) of various sizes occur between layers. In case of strong interlocking, the large shear stress and small deformations are registered. Conversely, if the interlocking is weak, the shear stress is small and the deformation is substantial,

- insufficiently bonded - the friction and bonding occur only as a result of the load and selfweight of layers.

As can be seen an appropriate bonding plays significant role in the functioning of the entire pavement structure and contributes to its quality and durability. Sufficiently bonded asphalt layers give improvement of the pavement structure strength and prevent surface deformation and cracks, as well as water penetration between asphalt layers [9].

There are a number of laboratory methods for determining the strength of bond between layers, but this type of research is limited in terms of the number of sampling points and the nature of information obtained. Among non-destructive tests, one could mention Impulse Hammer Test [10], the test using an FWD (Falling Weight Deflectometer) [11], or examinations with the use of acoustic methods [12].

Other very common non-destructive technique is the Ground Penetrating Radar method (GPR) [13-16]. This method is used routinely to assess the continuity of pavement layers, or for measuring the thickness of the asphalt structure. The GPR technique also offers a great potential in examining the detailed architecture of the asphalt packet and qualitative evaluation of the road's condition, including diagnoses of internal defects such as interlayer delaminations [17] or cracking [18]. These non-standard tasks are far more challenging as a study task, hidden with problems inherent in the nature and limitations of the method.

GPR measuring systems emit electromagnetic pulses towards the test object and receive the reflected signals. Emitted electromagnetic pulses passing through successive layers of materials with different dielectric properties are partly reflected on their boundaries. The primary recorded data is the return time and the amplitude of the signal reflected from the boundary.

The interpretation of echograms recorded is essential in the GPR methodology. One of the ways to identify the faulty interlayer connection was tested earlier using laboratory model and calculative examples [19]. This study presented methodology to identify the connections between layers of asphalt where delamination has occurred. This methodology is based on 
identifying the type of reflection, i.e. a single reflection $w(t)$ or double reflection $d w(t) / d t$, with one leading extreme or two adjacent leading extremes of inverse signs, respectively (Fig. 1). The simple single reflection is generated by the commonly known type of the planar boundary between a two homogeneous layers having mutual contrast of impedance. Depending on the sign of contrast the single reflection can be positive, as it is in case of boundary between successive mediums of low and high permittivity, or negative, as between mediums of high and low permittivity. When a crevice is very wide (in comparison with wavelength) the resulting signals are single type with positive or negative sign. When crevice becomes thinner and thinner the signal becomes similar to the derivative of the incident signal, its amplitude becomes proportional to the decreasing crevice thickness and then it is called the double reflection. The presence of strong positive double reflection marks the existence of serious damage at the joints of asphalt layers, e.g. delamination fissures filled with water. Strong negative double reflections may be the sign of sever dry delaminations, connections strongly sprayed with binder or cracks filled with foreign material of low permittivity.

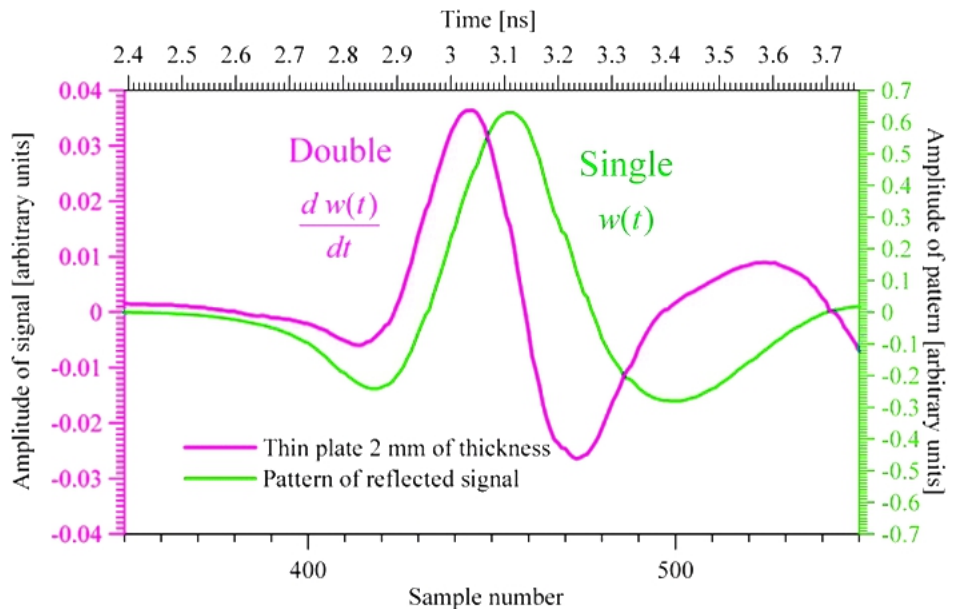

Fig. 1. Comparison of the head package shapes of the single $w(t)$ and the double reflection $d w(t) / d t$ measured on laboratory model of the crevice simulated by thin glass plate [9]

The double reflections have large diagnostic importance showing delaminations developed usually on the interlayer connections [20] and its amplitude can be a base of the effective thickness estimation, for example by use of direct waveform modeling. But in the field practice, a variety of complications should be expected when using this terminology like occurrence at mixed types of reflections and further complication of their shape in zones of substantial thickness (comparable to the local wavelength in the medium) and a gradient structure. Complications of the shape of reflections also occur as a result of diffraction where the pavement structure is characterized by the major local deviations from the plane-parallel structure. In the case of reflections of a small amplitude, serious difficulties of interpretation are also present in the identification of the type due to their observed shape being disturbed by masking background which is difficult to remove.

This paper presents the results of GPR surveys carried out on a road section where a problem with improper interlayer connection (bonding) between the layers of asphalt was found. The measurement was done using horn-type pulse antennae with centre frequencies of $2 \mathrm{GHz}$. Results showed a lack of layer connection in more than ten sections and sometimes over the entire width of the pavement. The article presents selected cases, confirmed by cores extracted at the specified locations. These cases are therefore an excellent illustration of the 
issue of recognizing the condition of interlayer connections in field practice. The term "interlayer connection" is used in this article instead of bonding just to underline the difference between laboratory and on test site evaluation of asphalt layer bonding.

According to the authors' opinion the following causes and phenomena, simultaneously present in the studied section of pavement are responsible for the lack of connection between the layers:

- insufficient compaction of upper layers; adverse weather conditions (wind and low temperature) during the laying of asphalt concrete in the base layers (dual layer) and the bonding layer, causing premature increase in viscosity of asphalt in the mix and hence hindrance to compaction; increased number of voids at the bottom of the layer (strong porosity of the floor of the top layer near the contact)

- no effect of interlock of contacting layers due to inefficient compaction of upper layer, - the segregation of the mixture in the upper layer; reduction of the contact area of layers due to the presence of clusters of coarse aggregate particles at the bottom of the top layer,

- lack of or inadequate spraying of the interlayer surface, the lack of bonding between layers, - increasing insufficiency of bond or an increase of the area of lack of bond between the layers resulting from the presence of water penetrating through micro fissures, insufficient compaction of the bottom layers, leaky longitudinal and transverse joints; intensified penetration of water due to interruption of the watercourse in the surrounding area,

- dirt in the interlayer space, failure to clean layers during the incorporation of successive layers.

The knowledge about these phenomena is very useful in explaining presence of strong, specific reflections on the recorded echograms. Opened delaminations, possibility of water penetration, presence of foreign material at the interlayer connection, porous base of the layer and change of particle size at the boundary have crucial importance in interpretation of the type, sign and amplitude of the resulting reflections.

\section{Field studies}

The results presented here are the effect of field studies carried out on one of the new sections of road where it passes through a deep trench with deficient hydrological conditions. GPR scans were taken along four parallel sections at four wheel tracks (two on each lane). The primary objective of this study was to perform a quantitative evaluation of the total thickness of layers built in, but the results also showed areas where the lack of a proper connection between layers of asphalt was found. The bad condition of the interlayer connection was confirmed by extracting cores at the specified locations of pavement.

Recorded echograms (Fig. 2) clearly show the layered structure of a packet of asphalt (wearing course, binding and base course asphalt layer). Even the very clear visibility of boundaries between the layers is intriguing, because the contrasts between the electrical properties of the lower layers of the packet (binding layer and base course) are usually minor and reflections corresponding to the boundaries should be hardly distinguishable from the remaining undeleted masking background. Significant contrast is present here only on the border between the wearing course and the binding layer. However, the phenomena occurring at interlayer interfaces in the course of applying layers lead to considerable material differences in the medium of layer near the interface (insufficient compaction, segregation or introduction of a foreign material). As a result, boundaries are often very well visible on echograms, but the corresponding reflections are rarely simple single reflections and their shape and amplitude reflect the complex structure of the contact. Thus, in interpretation practice, it is necessary to semi quantitatively evaluate the amplitude of reflections and to comment the amplitude using assumed properties of the medium and contact. 

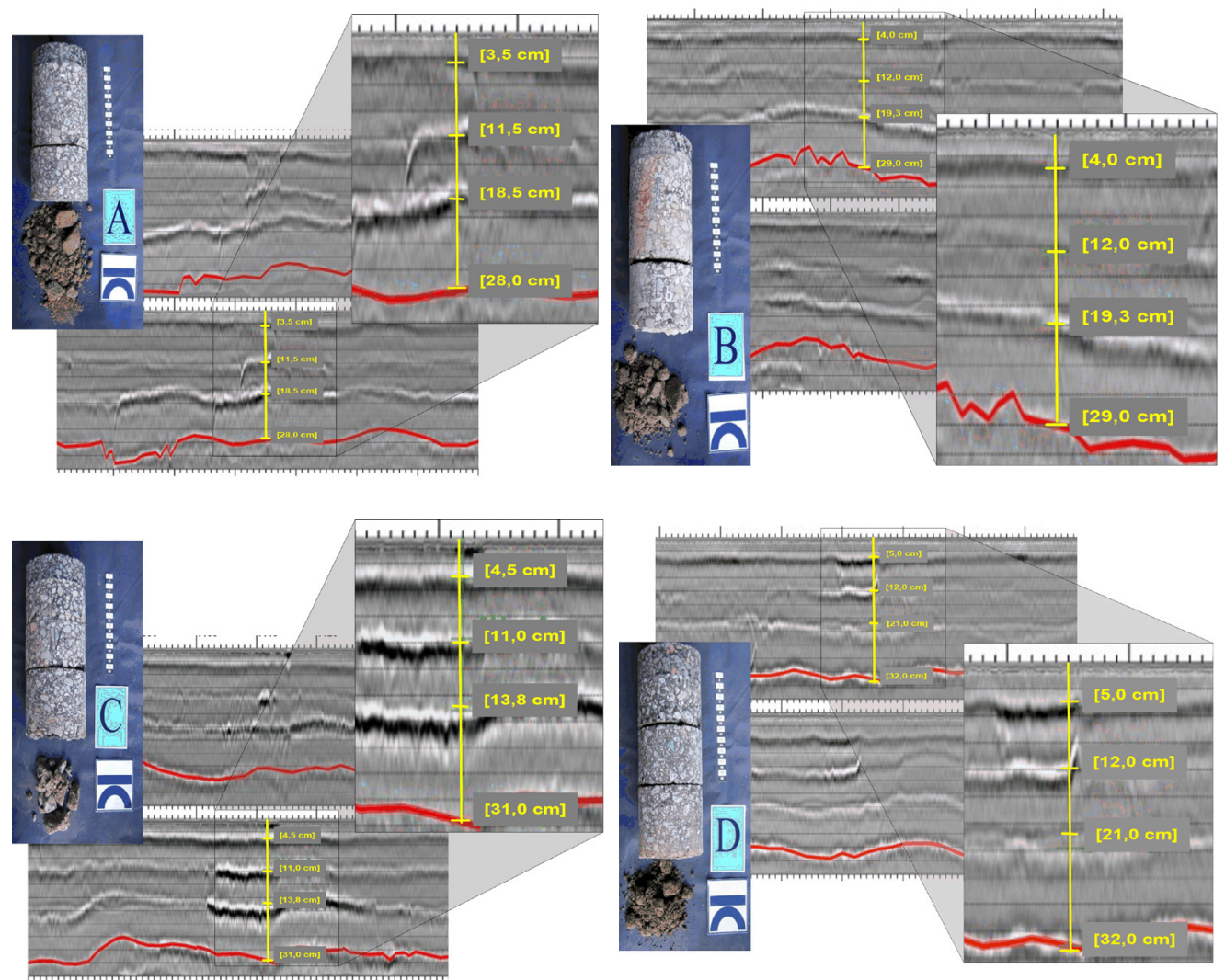

Fig. 2. Four sections (cases A, B, C and D) where the lack of a proper connection between asphalt layers was found. The cores ware taken at the specified locations of pavement on the basis of GPR echograms analyse. The yellow markings indicate the thicknesses of asphalt layers.

On the background of radar image of asphalt layers numerous, extremely strong positive double reflections are highlighted. At least four sites of particular accumulation of these strong reflections can be distinguished along the test sections. In addition to numerous local reflections, widespread reflections are also visible, occurring at lengths of several meters or even several tens of meters, often in all the profiles synchronously. Their depth position here always corresponds to interlayer surfaces, and their very large amplitude makes them stand out spectacularly from their environment in the adjacent areas of the profile. The positive sign of the double reflections and their high amplitude strongly suggest that they correspond to delamination fissures filled with water. Assuming a simple idealized model of such a fissure [9], and assuming its thickness $d$, it is possible to estimate very approximately the amplitude $\left.g_{R}{ }^{\text {Double }}\right|_{\max }$ of the corresponding double reflection:

$$
\left.\left.g_{R}^{\text {Double }}\right|_{\max } \approx \gamma \cdot 4 \pi \frac{d}{\lambda_{c}^{\prime}} \frac{r}{1-r^{2}} \cdot w_{I}\right|_{\max }
$$

Here $w_{l} l_{\max }$ is a characteristic amplitude of the incident signal, $r$ is the water-asphalt reflection coefficient, $\lambda_{c}^{\prime}$ is the central wavelength of the signal in water, $\gamma$ is an amplitude correcting factor corresponding to weakening of the signal as a result of the double transition through the road surface and geometrical divergence of the wave front. These estimates show 
that the strongest reflections in the test case correspond to the fissures, whose equivalent thickness $\mathrm{d}$ reaches up to $0.3 \mathrm{~mm}$.

The strongest extensive reflections are present in Case $\mathrm{C}$, in which hydrological problems are dramatically demonstrated at the roadside (Fig. 3a), and water infiltrated into the borehole from below (Fig. 3b).
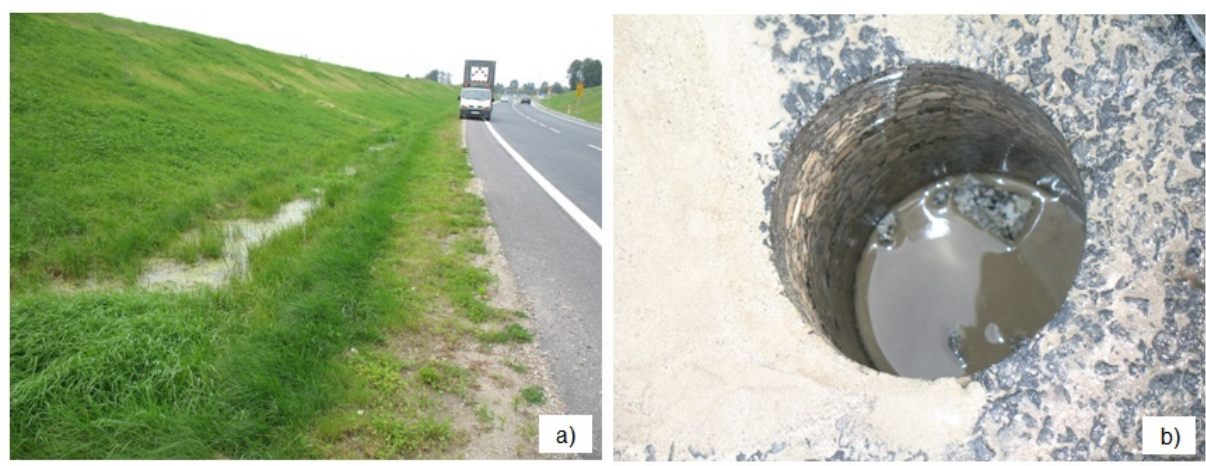

Fig. 3. a) section of road on which the drainage system is not functional; b) core extraction spot cleaned of dirt and water, spontaneously filling with water coming up from below.

Similarly, in case B there is a serious hydrological problem near the road. These observations are important because they confirm that water is the factor causing delamination cracks to show up in the echograms. Water will probably also intensify the development of defects, but as examination of cores demonstrated, the problematic interlayer connection has probably been present on the road ever since it was built. Strong double reflections probably correspond here to those places where the water entered (often extensively) into the faulty interfaces. The phenomenon of faulty interfaces (not necessarily wet), however, appears to be much more common. In echogram analysis and interpretation practice, it relates to clear visibility of interlayer horizons (large amplitude) and the type of the corresponding reflections, often more double (positive or negative) than single.

Careful quantitative analysis of layers is needed, especially in such cases described above. Observed changes in reflections, sometimes drastic (e.g. case D) do not necessarily indicate the same change in layer thickness. It should always be remembered that the radar echogram is a graphical presentation of the electromagnetic wave transition, the speed of which depends on the dielectric properties of the materials forming tested object.

\section{Summary}

The example of using GPR in pavement diagnosis, presented in this article, shows that this technique has great potential and described cases provide a very good illustration of diagnostic method in field conditions in terms of identifying pavement defects. Identification of interlayer connections at such early stage of road service often allows for more accurate planning of repairs, as well as seeking compensation payable under the guarantee by the contractor building the road.

A particular advantage of this method is the ability to identify and observe the subsurface damage. Sophisticated GPR observations of interlayer connection allows to evaluate bonding in more detailed way. Krysinski and Sudyka [9] describe response signals considering that boundaries of the interlayer connection are frequently not thin zones associated with a single or double reflection, but are usually thick, transitional zones manifested by the elongated response signals. Such blurred zones would correspond to: 
- undulated boundary surface,

- the belt where interlocking of the aggregate grains of the two contacting layers occurs,

- zone of changing porosity (e.g. insufficient compaction),

- zone of intense material degradation e.g. aggregate crumbling.

The most serious limitation of the method is caused by the difficulty in removing the background that masks the structural signals. Insufficiently effective removal of background, leads, among others, to significant problems in distinguishing a single reflection from a double one.

In the case of the investigated road the key phenomenon that cause a faulty connection, show up on an echogram (strong double positive reflection), is an intense infiltration of water into an empty or porous zone of interlayer connection. The additional phenomena at the interlayer connections investigated on echograms having evidence in the drilling cores are: - full delaminations between upper and lower base layer (sometimes between binding and base layer also),

- insufficient compaction at the base of binding layer and upper base layer (porous zones at the foot of these layers),

- presence of an foreign material at the connection.

\section{References}

1. P. Jaskula, 9th International Conference Environmental Engineering, 7 (2014)

2. C. Raab, M.N. Partl, Constr. Build. Mater. 23(8), 2926-2931 (2009)

3. S. Romanoschi, J. Metcalf, Transportation Res. Record 1778, 132-139 (2001)

4. J. Judycki, Drogownictwo, 275-279 (2003)

5. A.C. Raposeiras, D. Castro-Fresno, A. Vega-Zamanillo, J. Rodriguez-Hernandez, Constr. Build. Mater. 43, 372-381 (2013)

6. A. Vaitkus, D. Žilionienè, S. Paulauskaitè, F. Tuminienè, L. Žiliūtè, The Baltic J. of Road and Bridge Eng. 6, 210-218 (2011)

7. A.C. Raposeiras, A. Vega-Zamanillo, M.A. Calzada-Pérez, D. Castro-Fresno, Constr. Build. Mater. 44, 228-235 (2013)

8. A. Vaitkus, D. Cygas, A. Laurinavicius, V. Vorobjovas, R. Kleiziene, Građevinar 64, 915-920 (2012)

9. J. Sudyka, L. Krysiński, Int. J. Pavement Res. Technol. 4(3), 176-184 (2011)

10. C. Sangiorgi, A.C. Collop, N.H. Thom, International Symposium: Non-Destructive Testing in Civil Engineering (2008)

11. B. Al Hakim, L.W. Cheung, R.J. Armitage, Int. J. Pavement Eng. 1, 49-59 (1999)

12. A. Saeed, J.W. Hall, Pavement evaluation 601 (2002)

13. L. Laguerre, L. Laguerre, F. Liu, F. Liu, O. Durand, O. Durand, X. Dérobert, X. Dérobert, Symp. A Q. J. Mod. Foreign Lit., 26-28 (200)

14. J.J. Daniels, Ground Penetrating Radar Fundamentals (USEPA Publ., 2000)

15. S. Lahouar, I.L. Al-Qadi, NDT and E International 41, 69-81 (2008)

16. X. Dérobert X, Ground Penetrating Radar 2nd Edition (J. Daniels, Ed. 2009)

17. L.F. Walubita, T. Scullion, J. Leidy, W. Liu, Road Mater. Pav. Des. 10, 259-286 (2009)

18. L. Krysiński, J. Sudyka, J. Appl. Geophys. 97, 27-36 (2013)

19. L. Krysiński, J. Sudyka, Nondestruct. Test. Eval. 27(May), 219-227 (2012) 
20. A. Van Der Wielen, L. Courard, F. Nguyen, Proceedings of the 13th International Conference on Ground Penetrating Radar (GPR 2010, 2010) 